\title{
Spin-stretching modes in anisotropic magnets: spin-wave excitations in the multiferroic $\mathrm{Ba}_{2} \mathrm{CoGe}_{2} \mathrm{O}_{7}$
}

\author{
K. Penc, ${ }^{1,2}$ J. Romhányi, ${ }^{1,2}$ T. Rõõm, ${ }^{3}$ U. Nagel,${ }^{3}$ Á. Antal, ${ }^{2}$ T. Fehér, ${ }^{2}$ A. Jánossy, ${ }^{2}$ H. \\ Engelkamp, ${ }^{4}$ H. Murakawa, ${ }^{5,6}$ Y. Tokura,,${ }^{5,6,7,8}$ D. Szaller, ${ }^{2}$ S. Bordács, ${ }^{2,5,6}$ and I. Kézsmárki ${ }^{2,5}$ \\ ${ }^{1}$ Institute for Solid State Physics and Optics, Wigner Research Centre for Physics, \\ Hungarian Academy of Sciences, H-1525 Budapest, P.O.B. 49, Hungary \\ ${ }^{2}$ Department of Physics, Budapest University of Technology and Economics and Condensed \\ Matter Research Group of the Hungarian Academy of Sciences, 1111 Budapest, Hungary \\ ${ }^{3}$ National Institute of Chemical Physics and Biophysics, 12618 Tallinn, Estonia \\ ${ }^{4}$ High Field Magnet Laboratory, Institute for Molecules and Materials, \\ Radboud University, 6525 ED Nijmegen, The Netherlands \\ ${ }^{5}$ Multiferroics Project, ERATO, Japan Science and Technology Agency (JST), \\ Japan c/o The University of Tokyo, Tokyo 113-8656, Japan \\ ${ }^{6}$ Quantum-Phase Electronics Center, Department of Applied Physics, \\ The University of Tokyo, Tokyo 113-8656, Japan \\ ${ }^{7}$ Department of Applied Physics, The University of Tokyo, Tokyo 113-8656, Japan \\ ${ }^{8}$ Cross-correlated materials group (CMRG) and correlation electron research group (CERG), \\ RIKEN Advanced Science Institute, Wako 351-0198, Japan
}

(Dated: August 24, 2018)

\begin{abstract}
We studied spin excitations of the non-centrosymmetric $\mathrm{Ba}_{2} \mathrm{CoGe}_{2} \mathrm{O}_{7}$ in high magnetic fields up to $33 \mathrm{~T}$. In the electron spin resonance and far infrared absorption spectra we found several spin excitations beyond the two conventional magnon modes expected for such a two-sublattice antiferromagnet. We show that a multi-boson spin-wave theory describes these unconventional modes, including spin-stretching modes, characterized by oscillating magnetic dipole and quadrupole moment. The lack of the inversion symmetry allows each mode to become electric dipole active. We expect that the spin-stretching modes can be generally observed in inelastic neutron scattering and light absorption experiments in a broad class of ordered $S>1 / 2$ spin systems with strong single-ion anisotropy and/or non-centrosymmetric lattice structure.
\end{abstract}

PACS numbers: $75.85 .+\mathrm{t}$ 75.30.Gw 75.10.-b 76.50.+g

Magnons are collective spin excitations in crystals with long-range magnetic order, often investigated by electromagnetic absorption and neutron scattering experiments. Both classical and quantum spin-wave theory of $S=1 / 2$ systems predict one magnon branch in the spin-excitation spectrum for each spin in the magnetic unit cell [1]. This rule about the number of magnon branches is generally accepted and experimentally verified for $S>1 / 2$ spin systems as long as the conventional spin-wave theory applies, requiring that the lengths (i.e., the absolute values of the expectation values) of the spins are preserved in the excited states and only their orientations change relative to the ground-state configuration [2]. However, the picture of one magnon mode per spin in the magnetic unit cell needed to be surpassed in several $f$-electron compounds with complicated quadrupolar ordering, such as $\mathrm{CeB}_{6}[3]$ and $\mathrm{UO}_{2}[4]$.

Recently, additional spin-wave modes have been observed by far infrared (FIR) spectroscopy [5] and inelastic neutron scattering (INS) [6] in $\mathrm{Ba}_{2} \mathrm{CoGe}_{2} \mathrm{O}_{7}$, a simple two-sublattice easy-plane antiferromagnet (AF) with $S=3 / 2$ spins $[7,[8]$. This material has attracted much interest owing to its multiferroic ground state where delicate magnetic control of the ferroelectric polarization [8, 9] and chirality [10] were realized. Moreover, spin waves in $\mathrm{Ba}_{2} \mathrm{CoGe}_{2} \mathrm{O}_{7}$ exhibits giant directional dichroism and natural optical activity at $\mathrm{THz}$ frequencies due to the large ac magnetoelectric effect [5, 10]. A recent numerical diagonalization study on finite spin clusters found, besides the two conventional AF modes, additional spin resonances with peculiar optical properties [10, 11]. Nevertheless, the understanding of the unconventional magnon modes and the coupled dynamics of spins and electronic polarization on a fundamental level remained an open issue.

In this Letter, we investigate the spin-wave excitations in $\mathrm{Ba}_{2} \mathrm{CoGe}_{2} \mathrm{O}_{7}$ over a broad photon energy range combining electron spin resonance (ESR) and high-resolution FIR spectroscopy. The largest magnetic field, $33 \mathrm{~T}$, applied in this study drastically changes the antiferromagnetic spin configuration for any field direction, in contrast to former experiments restricted to $B_{\mathrm{dc}} \leq 12 \mathrm{~T}$. The orientation of $B_{\mathrm{dc}}$ and the light polarization relative to the main crystallographic axes were systematically varied in order to map the field dependence and the selection rules of the modes. We derive a multi-boson spin-wave theory and show that a large single-ion anisotropy plays a key role in the emergence of new magnetic excitations involving the oscillation of spin length, and that the lack of inversion symmetry, a necessary condition for the dc 

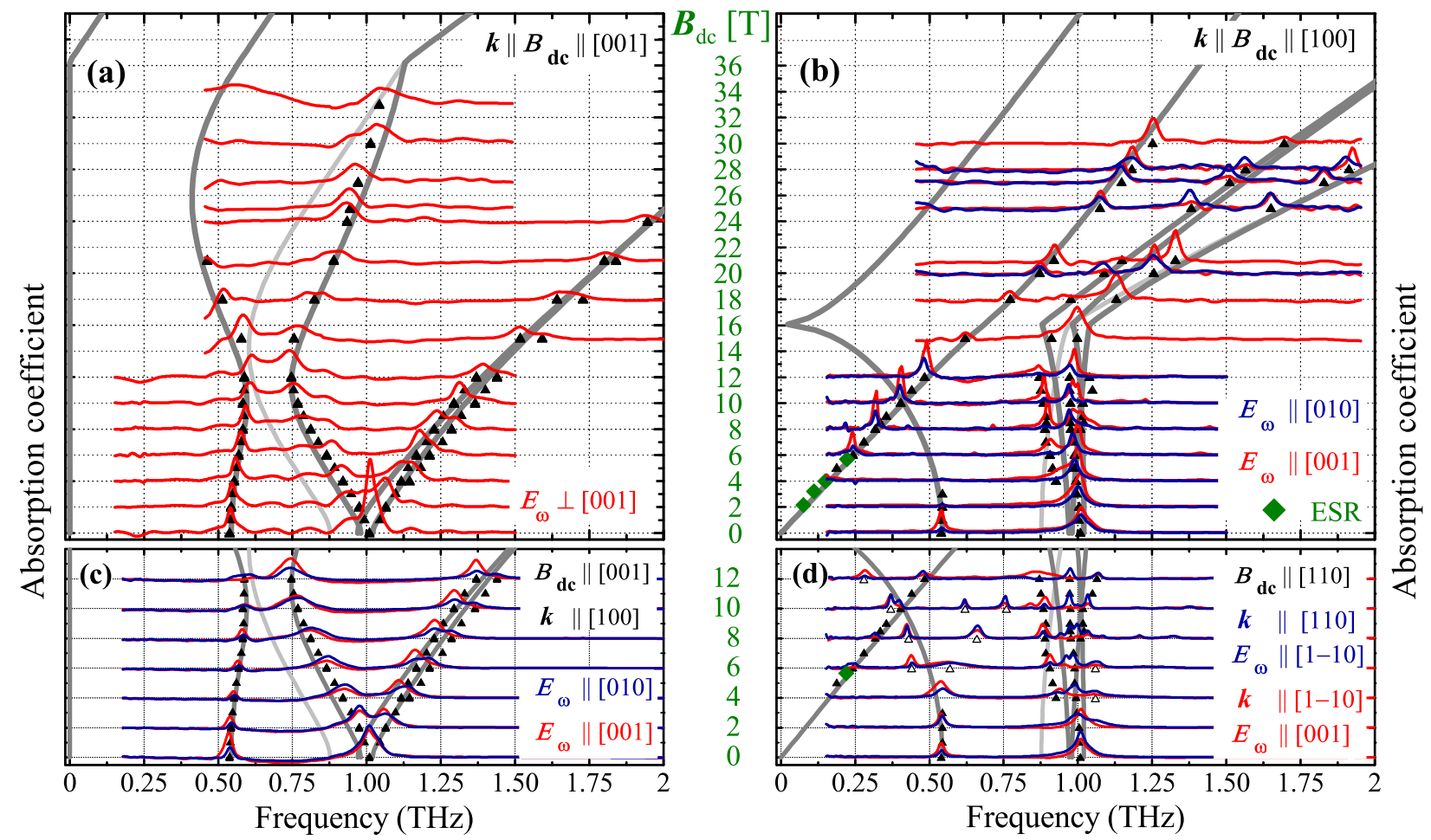

FIG. 1. (color online). Magnetic field dependence of the absorption spectra in $\mathrm{Ba}_{2} \mathrm{CoGe}_{2} \mathrm{O}_{7}$ below 2 THz for a representative set of light polarizations. The spectra are shifted vertically proportional to the magnitude of the field, $B_{\mathrm{dc}}$. The distance between horizontal grid lines corresponds to $20 \mathrm{~cm}^{-1}$ in panels (a) and (c), and $30 \mathrm{~cm}^{-1}$ in (b) and (d). The direction of $B_{\mathrm{dc}}$ is indicated in each panel and the spectra for different polarizations and propagation directions (k) of light are distinguished by the color. Black triangles and green diamonds represent the position of the resonances determined from the FIR and ESR spectra, respectively. The grey lines show the field dependence of the modes obtained in our multi-boson spin-wave approach. (d) For $B_{\mathrm{dc}} \perp$ [001] in some polarization configurations we observed additional modes (open triangles) that are not explained by the theory. Two cases $(E \|[1 \overline{1} 0]$ and $E \|[001])$ are shown here.

and ac magnetoelectric effects, renders these spin-waves electric-dipole active.

$\mathrm{Ba}_{2} \mathrm{CoGe}_{2} \mathrm{O}_{7}$ has a non-centrosymmetric tetragonal space group, $\mathrm{P} \overline{4} 2_{1} m$. The magnetic $\mathrm{Co}^{2+}$ ions are surrounded by tetrahedra of oxygens compressed along the [001] tetragonal axis. Due to the lack of inversion symmetry a coupling between spins and local polarization appears [12, 13]. This was observed as a magnetic-order induced ferroelectricity in this family of materials including $\mathrm{Ba}_{2} \mathrm{CuGe}_{2} \mathrm{O}_{7}$ [8, 9], $\mathrm{Ca}_{x} \mathrm{Sr}_{2-x} \mathrm{CoSi}_{2} \mathrm{O}_{7}$ [14], and $\mathrm{Ba}_{2} \mathrm{MnGe}_{2} \mathrm{O}_{7}$ [15].

Here we study spin-wave resonances of $\mathrm{Ba}_{2} \mathrm{CoGe}_{2} \mathrm{O}_{7}$ on high-quality single crystals [8] in the magnetic phase at $T=3.5 \mathrm{~K}$. ESR spectroscopy was performed at 75 , 111, 150, and $222 \mathrm{GHz}$ using solid state oscillators, while FIR transmission was measured by Fourier transform spectroscopy over the region of $0.15-2 \mathrm{THz}(0.6-8 \mathrm{meV})$ with a resolution of $15 \mathrm{GHz}$.

An overview of the spectra for representative directions of the magnetic field is given in Fig. 1. Two sharp peaks are present in the zero-field FIR absorption spectra at $f \sim 0.5$ and $1 \mathrm{THz}$, in accordance with former studies $[5$, 10]. The first is assigned to the usual optical magnon branch gapped by magnetic anisotropy of mostly singleion origin. The second is not captured by conventional spin-wave theory, and was shown to respond to both the magnetic and electric component of light and termed as an electromagnon [5]. For $B_{\mathrm{dc}}$ along the tetragonal axis as in Fig. 1(a) and (c), the $1 \mathrm{THz}$ mode shows a V-shape splitting with a double-peak structure on the high-energy side. The double-peak structure is clearly visible when $E_{\omega} \|$ [010] [see the blue curves in Fig. 1(c)]. The frequency of the $0.5 \mathrm{THz}$ mode slightly increases in low fields [Fig. 1(a) and (c)], however, it turns back after an avoided crossing with the lower branch of the $1 \mathrm{THz}$ resonance at $B_{\mathrm{dc}} \approx 12 \mathrm{~T}$.

The rotation of $B_{\mathrm{dc}}$ from the tetragonal axis to the tetragonal plane affects all the modes drastically (Fig. (1). For $B_{\mathrm{dc}} \|[100]$, the $1 \mathrm{THz}$ mode is again split into three distinct lines. However, they exhibit only a weak softening up to a kink at $B_{\mathrm{dc}} \approx 16 \mathrm{~T}$, from where the resonance frequencies start to increase quickly [Fig. 1(b)]. The magnon mode at $f \sim 0.5 \mathrm{THz}$ becomes silent with increasing field for both polarization directions in the Faraday geometry $\left(\mathbf{k} \| B_{\mathrm{dc}}\right)$.

An additional low-frequency mode appears in the ESR 
and FIR spectra when $B_{\mathrm{dc}}$ is within the tetragonal plane, breaking the fourfold rotoinversion symmetry of the lattice. This mode corresponds to the quasi-Goldstone mode of an easy-plane AF when $B_{\mathrm{dc}}=0$. The frequency of this mode is not affected measurably by the orientation of $B_{\mathrm{dc}}$ in the plane and follows a linear field dependence down to $75 \mathrm{GHz}$. Hence, the in-plane anisotropy gap is less than $75 \mathrm{GHz}$.

If $B_{\mathrm{dc}}$ is in the tetragonal plane, the number of observed resonances exceeds six in some polarization configurations. Two representative cases are presented in Fig. 1(d). The $0.5 \mathrm{THz}$ mode suddenly splits into a sharp and a broad feature at $B_{\mathrm{dc}}=5 \mathrm{~T}$, while the $1 \mathrm{THz}$ branch consists of at least four resonances. At $\gtrsim 12 \mathrm{~T}$, the number of modes is reduced.

As a microscopic model, we consider the Hamiltonian below to describe the $S=3 / 2$ spin $\mathrm{Co}^{2+}$ ions. Following Ref. 11 , we have a large single-ion anisotropy $\Lambda$, but we introduce an anisotropic exchange coupling $(J$ and $J_{z}$ ) and neglect the Dzyaloshinskii-Moriya term that appeared to have negligible effect on the excitations:

$$
\begin{aligned}
\mathcal{H}= & J \sum_{\langle i, j\rangle}\left(S_{i}^{x} S_{j}^{x}+S_{i}^{y} S_{j}^{y}\right)+J_{z} \sum_{\langle i, j\rangle} S_{i}^{z} S_{j}^{z}+ \\
& \sum_{i}\left[\Lambda\left(S_{i}^{z}\right)^{2}+g_{z z} h_{z} S_{i}^{z}+g_{x x}\left(h_{x} S_{i}^{x}+h_{y} S_{i}^{y}\right)\right]
\end{aligned}
$$

where $\langle i, j\rangle$ indicates nearest neighbor pairs, and the $x$, $y$, and $z$ axes are parallel to the [110], [1]10], and [001] crystallographic directions, respectively. $g_{x x}=g_{y y}$ and $g_{z z}$ are the principal values of the $g$ tensor, and $h_{\alpha}=$ $\mu_{\mathrm{B}} B_{\mathrm{dc}, \alpha}$ are the components of the magnetic field.

We assume a site-factorized variational wave function $\left|\Psi_{0}\right\rangle=\prod_{i \in A}\left|\Psi_{A}(i)\right\rangle \prod_{i \in B}\left|\Psi_{B}(i)\right\rangle$ to describe the longrange ordered ground state of two spin sublattices $A$ and $B$ ( $i$ is the site index). For example, when $B_{\mathrm{dc}} \|[110]$ and $J_{z} / J \lesssim 4$, we get a canted Néel state [16] where the expectation values of spin components are

$$
\left\langle\Psi_{X}(i)\left|\hat{\mathbf{S}}_{X}\right| \Psi_{X}(i)\right\rangle=\frac{3 \eta(\eta+1)}{3 \eta^{2}+1}\left(\cos \varphi_{X}, \sin \varphi_{X}, 0\right) .
$$

The two variational parameters $\eta$ and $\varphi_{A}=-\varphi_{B}$ are determined from the minimization of the energy $(X=$ $A, B)$. We note that $\eta \neq 1$ corresponds to a spin with length smaller than $3 / 2$, the consequence of the on-site anisotropy.

This $\left|\Psi_{0}\right\rangle$ serves as a starting point to study excitations: we introduce four orthogonal bosons on each site, denoted by $a_{\nu, X}^{\dagger}(i)$, where $\nu=0, \ldots, 3$, so that the variational ground state is $\left|\Psi_{X}(i)\right\rangle=a_{0, X}^{\dagger}(i) \mid$ vacuum $\rangle$. Any product of the operators on a site can be expressed as a quadratic form of the four $a$ bosons and they satisfying the expected commutation relations. The number of bosons on each site is conserved, $\sum_{\nu=0}^{3} a_{\nu, X}^{\dagger} a_{\nu, X}=M$, and $M=1$ for the $S=3 / 2$ spin. The linear flavor-wave theory is a $1 / M$ expansion, where the $a_{\nu, A}^{\dagger}$ and $a_{\nu, B}^{\dagger}$ with
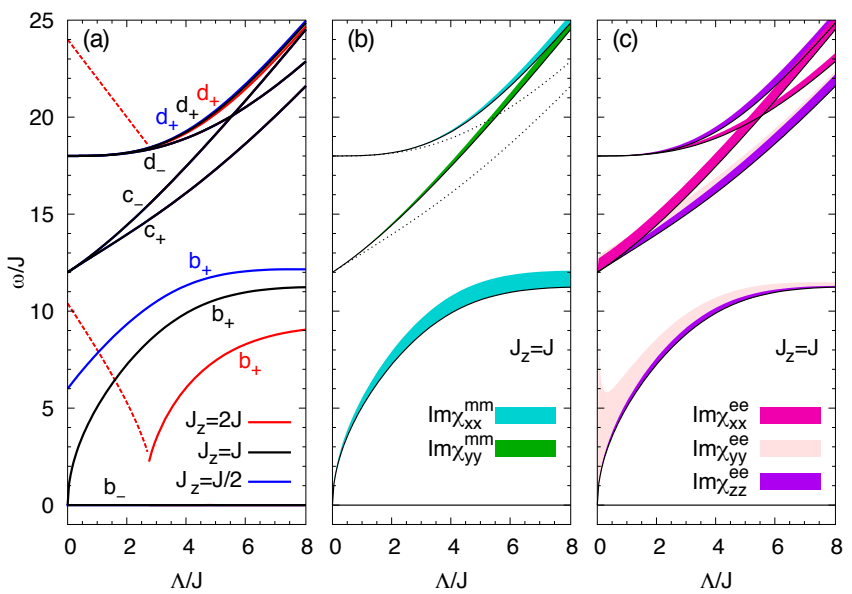

FIG. 2. (color online). (a) The energy of the modes for different values of $J_{z} / J$ in zero field. $b_{-}$denotes the $\omega=0$ Goldstone mode. Only the $b_{+}$and $d_{+}$modes depend on $J_{z} / J$. The dashed lines indicate modes in the easy-axis AF state that forms below $\Lambda \approx 2.7 J$ for $J_{z}=2$. (b) and (c) shows the imaginary part of the magnetic $\chi_{\xi \xi}^{m m}(\omega)$ and electric $\chi_{\xi \xi}^{e e}(\omega)$ dynamic susceptibilities, respectively, for $J=J_{z}$. The shading above the lines represent the strength of the magnetic and electric response.

$\nu=1,2,3$ play the role of the Holstein-Primakoff bosons and describe the excitations, the generalized spin-waves. Replacing $a_{0, X}^{\dagger}$ and $a_{0, X}$ with $\left(M-\sum_{\nu=1}^{3} a_{\nu, X}^{\dagger} a_{\nu, X}\right)^{1 / 2}$ and performing the expansion in $1 / M$ one can follow the procedure of the conventional spin-wave theory, and we get a Hamiltonian that is quadratic in boson operators and straightforward to diagonalize [17]. A similar approach has been used to describe, e.g., $\mathrm{CeB}_{6}$ [3], the $\mathrm{SU}(4)$ Heisenberg model [18], the $\mathrm{TlCuCl}_{3}$ spin ladder [19], and multipolar excitations in the spin-1 20] and spin-3/2 [21] Heisenberg models.

The spectrum as a function of $\Lambda / J$ in zero magnetic field and for zero momentum is shown in Fig. 2(a). It consists of six modes, three for each Co spin in the unit cell. A finite anisotropy reduces the $\mathrm{O}(3)$ symmetry of the Hamiltonian to $\mathrm{O}(2)$, decreasing the number of zero energy Goldstone modes from two to one.

Let us begin from $\Lambda=0$. Then $\eta=1$ and the $a_{0, X}^{\dagger}$ creates a spin coherent state with maximal spin length $3 / 2$. In this limit the $b_{ \pm}$branches correspond to magnons of the standard spin-wave theory and they are decoupled from the other modes. The $c_{ \pm}$and $d_{ \pm}$are local magnetic transitions with $\Delta S_{W}=2$ and 3 corresponding to Zeemann energies $12 J$ and $18 J$, respectively, in the Weiss field $4 \times(3 / 2) J$ of the neighboring spins $\left(S_{W}\right.$ is the spin component parallel to the Weiss field). The $c_{ \pm}$and $d_{ \pm}$modes are generally silent in neutron, ESR, and FIR spectra, as the magnetic dipolar matrix elements vanish in the imaginary part of the dynamic magnetic susceptibility, $\operatorname{Im} \chi_{\alpha \alpha}^{m m}(\omega) \propto \sum_{f}\left|\left\langle f\left|S^{\alpha}\right| \Psi_{0}\right\rangle\right|^{2} \delta\left(\omega-\omega_{f}+\omega_{0}\right)$. 

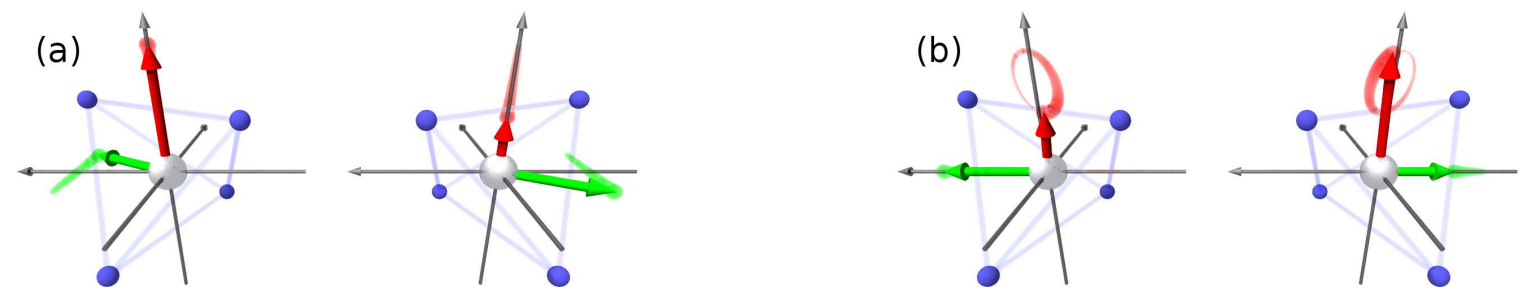

FIG. 3. (color online). Motion of the magnetizations (green arrows) and the local electric polarizations (red arrows) in the two sublattices (a) for the Goldstone mode ( $b_{-}$in Fig. 2) and (b) for the $c_{-}$stretching mode. The blue spheres are the oxygens forming tetrahedral cages around the central Co ions. The vertical axis is the tetragonal one, while the horizontal axes point along [110] and [110]. Apparent tilting of the axes comes from the perspective view.

These transitions can only be excited by quadrupolar or higher order spin operators.

As we turn on $\Lambda>0, \eta$ increases and the spin length decreases in the Néel ground state [Eq. (2)]. The modes labeled as $c_{-}$and $c_{+}$in Fig. 2 are spin-stretching modes, with spin length oscillating in and out of phase on the two sublattices, respectively. Hence $c_{-}$is excited by the $S^{y}$ spin operator, with a finite weight in $\operatorname{Im} \chi_{y y}^{m m}(\omega)$ that vanishes as $(\Lambda / J)^{2}$ when $\Lambda / J \rightarrow 0$. Most of the weight in $\operatorname{Im} \chi_{x x}^{m m}(\omega)$ comes from the low-energy $b_{+}$mode, while the contribution of $d_{+}$to $\operatorname{Im} \chi_{x x}^{m m}(\omega)$ is $\propto(\Lambda / J)^{4}$, so that the sum rule $\int d \omega \operatorname{Im} \chi_{x x}^{m m}(\omega) / \omega=g_{x x}^{2} / 8 J$ is fulfilled. $\operatorname{Im} \chi_{z z}^{m m}(\omega)$ is zero for all but the Goldstone mode $b_{-}$.

For a large on-site anisotropy $\left(\Lambda \gg J, J_{z}\right), \eta \rightarrow \Lambda / 3 J$ in the leading order and the $S^{z}= \pm 3 / 2$ states are suppressed in the ground state, reducing the spin length to 1 . We recover the spectra of isolated spins with single-ion anisotropy: two modes with energies $\omega / \Lambda \rightarrow 0$ and four modes with $\omega \rightarrow 2 \Lambda$, in agreement with Ref. 11 .

From the analysis of the dynamic magnetic susceptibility it follows that these unconventional spin excitations become observable by ESR, FIR, and neutron scattering as soon as the single-ion anisotropy gets significant. Moreover, if the crystal lattice breaks the inversion symmetry, spin quadrupolar and electric dipole (or electric polarization) operators have the same symmetry properties. Thus, a new channel opens to excite these modes as the electric field of the incident light can directly couple to the spin quadrupolar operators, response expressed by the $\operatorname{Im} \chi_{\alpha \alpha}^{e e}(\omega)$ [11]. Indeed, $d_{-}$and $c_{+}$modes with only magnetic quadrupol moment are excited this way and remain silent in $\operatorname{Im} \chi_{\alpha \alpha}^{m m}(\omega)$ irrespective of the $\Lambda / J$ ratio. The dynamical electric susceptibility $\operatorname{Im} \chi_{\alpha \alpha}^{e e}(\omega)$ shows a strong response for most of the modes, as shown in Fig. 2(c).

Our model describes well the magnetic field dependence of the spin-wave frequencies in $\mathrm{Ba}_{2} \mathrm{CoGe}_{2} \mathrm{O}_{7}$, see Fig. 1 From the fit of the experimental data we obtain $\Lambda=13.4 \mathrm{~K}, J=2.3 \mathrm{~K}, J_{z}=1.8 \mathrm{~K}, g_{z z}=2.1$ and $g_{x x}=g_{y y}=2.3$. Magnetic field larger than $16 \mathrm{~T}$ in the easy plane is strong enough to drive a transition from a canted $\mathrm{AF}$ to an almost saturated magnet. This is observed as a kink in the $1 \mathrm{THz}$ modes at $B_{\mathrm{dc}} \approx 16 \mathrm{~T}$
[Fig. 1(b)]. The theory also predicts the onset of fully saturated phase for $B_{\mathrm{dc}}>36 \mathrm{~T}$ applied perpendicular to the easy plane (along the tetragonal axis), inducing a gap in the Goldstone mode [Fig. 1(a)], in agreement with Ref. 22. The V-shape splitting of the $1 \mathrm{THz}$ mode and the avoided crossing at $B_{\mathrm{dc}} \approx 12 \mathrm{~T}$ for fields parallel to the tetragonal axis is also reproduced correctly [see Fig. 1(a) and (c)]. The lowest-lying mode of the $f \sim 1 \mathrm{THz}$ branch is theoretically predicted to be weak [see dotted grey line in Fig. 2(b)] and does not appear in the experimental spectra. The only feature not explained by the model is the splitting of the $f \sim 0.5 \mathrm{THz}$ resonance above $B_{\mathrm{dc}}=5 \mathrm{~T}$ for fields perpendicular to the [001] axis.

The analytical solution of a pure spin Hamiltonian [see Eq. (1)] enabled us to fully characterize the excited states in terms of spin and polarization dynamics, implying that the electric polarization adiabatically follows the sublattice magnetization vector and does not have its "own" dynamics in the energy range of interest. The motion of the sublattice magnetization and the local polarization in zero field is visualized in Fig. 3 for the Goldstone mode and for a spin-stretching mode. The Goldstone mode is associated with the oscillation of the polarization along the tetragonal axis and has a direct connection with the dc magnetoelectric effect. The spin-stretching mode shows more complex polarization dynamics [23], e.g., for $\Lambda \rightarrow 0$ the polarization still oscillates even though the magnetic moment is frozen.

Our theory describes the unconventional spin-wave excitations in $\mathrm{Ba}_{2} \mathrm{CoGe}_{2} \mathrm{O}_{7}$ and provides a guide for spinwave spectroscopy in a broad class of ordered magnets with strong magnetic anisotropy and/or a noncentrosymmetric lattice structure. We expect unconventional spin excitations to emerge in the dynamic magnetic susceptibility whenever a large single-ion anisotropy is present in a $S>1 / 2$ system. Moreover, if the inversion symmetry of the crystal is broken, these new modes can have a dielectric response even in the absence of magnetic anisotropy. They should be detected by THz light absorption or inelastic neutron scattering via the induced magnetic and/or electric dipole moment.

We are grateful for stimulating discussions with 
S. Miyahara, N. Furukawa, N. Kida, Y. Onose, and B. Náfrádi. This work was supported by Hungarian OTKA under Grant Nos. PD75615, CNK80991, K73361, K73455, and NN76727, Bolyai program, TÁMOP4.2.1/B-09/1/KMR-2010-0002, by EuroMagNET II under the EU contract number 228043, by Estonian Ministry of Education and Research Grant SF0690029s09, Estonian Science Foundation Grants ETF8170 and ETF8703, and the bilateral programme of the Estonian and Hungarian Academies of Science.

[1] J. Van Kranendonk and J. H. Van Vleck, Rev. Mod. Phys. 30, 1 (1958).

[2] E. Turov, Physical Properties of Magnetically Ordered Crystals (Academic Press, New York, 1965).

[3] R. Shiina, H. Shiba, P. Thalmeier, A. Takahashi, and O. Sakai, Journal of the Physical Society of Japan 72, 1216 (2003).

[4] S. Carretta, P. Santini, R. Caciuffo, and G. Amoretti, Phys. Rev. Lett. 105, 167201 (2010),

[5] I. Kézsmárki, N. Kida, H. Murakawa, S. Bordács, Y. Onose, and Y. Tokura, Phys. Rev. Lett. 106, 057403 (2011).

[6] C. de la Cruz, private communication.

[7] A. Zheludev, T. Sato, T. Masuda, K. Uchinokura, G. Shirane, and B. Roessli, Phys. Rev. B 68, 024428 (2003).

[8] H. Murakawa, Y. Onose, S. Miyahara, N. Furukawa, and Y. Tokura, Phys. Rev. Lett. 105, 137202 (2010).

[9] H. T. Yi, Y. J. Choi, S. Lee, and S.-W. Cheong, Appl. Phys. Lett. 92, 212904 (2008)

[10] S. Bordacs, I. Kezsmarki, D. Szaller, L. Demko, N. Kida, H. Murakawa, Y. Onose, R. Shimano, T. Room, U. Nagel, S. Miyahara, N. Furukawa, and Y. Tokura, ArXiv eprints (2011), arXiv:1109.1597 [cond-mat.str-el].

[11] S. Miyahara and N. Furukawa, Journal of the Physical Society of Japan 80, 073708 (2011)

[12] P. Toledano, D. D. Khalyavin, and L. C. Chapon, Phys. Rev. B 84, 094421 (2011)

[13] K. Yamauchi, P. Barone, and S. Picozzi, Phys. Rev. B 84, 165137 (2011)

[14] M. Akaki, J. Tozawa, D. Akahoshi, and H. Kuwahara, Applied Physics Letters 94, 212904 (2009). M. Akaki, J. Tozawa, M. Hitomi, D. Akahoshi, and H. Kuwahara, Journal of Physics: Conference Series 200, 012003 (2010)

[15] H. Murakawa, private communication.

[16] J. Romhányi, F. Pollmann, and K. Penc, Phys. Rev. B 84, 184427 (2011) J. Romhányi, M. Lajkó, and K. Penc, Phys. Rev. B 84, 224419 (2011)

[17] See supplementary material at [URL] for the exact form of the transformed Hamiltonian and the details of the calculation.

[18] A. Joshi, M. Ma, F. Mila, D. N. Shi, and F. C. Zhang, Phys. Rev. B 60, 6584 (1999).

[19] M. Matsumoto, B. Normand, T. M. Rice, and M. Sigrist, Phys. Rev. B 69, 054423 (2004)

[20] N. Papanicolaou, Nuclear Physics B 240, 281 (1984) Nuclear Physics B 305, 367 (1988)

[21] F. Onufrieva, Zhurnal Eksperimentalnoi i Teoretich- eskoi Fiziki 89, 2270 (1985); A. V. Chubukov, Journal of Physics: Condensed Matter 2, 1593 (1990).

[22] J. W. Kim, S. H. Khim, S. H. Chun, K. H. Kim, E. Choi, Y. Jo, L. Balicas, N. Harrison, H. Yi, S.-W. Cheong, J. H. Han, and C. D. Batista, Investigation of a Quantum Critical Point in Multiferroic $\mathrm{Ba}_{2} \mathrm{CoGe}_{2} \mathrm{O}_{7}$, Annual Report p. 20 (National High Magnetic Field Laboratory, 2010).

[23] See supplementary material at [URL] for an animated representation of each mode. 


\section{SUPPLEMENT}

In this supplement we show in more details the multiboson spin-wave theory. For simplicity, we consider the case when the magnetic field is in the easy plane. For magnetic field perpendicular to the easy-plane the expressions become rather complicated, and the energy of the modes that was shown in Fig. 1 can be obtained numerically.

\section{Bosonic representation of the spin operators}

First we introduce the bosons $\alpha_{m}^{\dagger}$ that create the $S^{z}=m$ states of the $S=3 / 2$ spin, i.e. $|m\rangle=$ $\alpha_{m}^{\dagger} \mid$ vacuum $\rangle$. The number of bosons on each site is con- served, $\sum_{m} \alpha_{m}^{\dagger} \alpha_{m}=M$, and $M=1$ for the $S=3 / 2$ spin. Using the four $\alpha$ bosons, the spin operators can all be expressed as quadratic forms, for example

$$
\begin{aligned}
S^{z} & =\sum_{m=-3 / 2}^{3 / 2} m \alpha_{m}^{\dagger} \alpha_{m} \\
\left(S^{z}\right)^{2} & =\sum_{m=-3 / 2}^{3 / 2} m^{2} \alpha_{m}^{\dagger} \alpha_{m} \\
S^{+} & =\sqrt{3}\left(\alpha_{3 / 2}^{\dagger} \alpha_{1 / 2}+\alpha_{-1 / 2}^{\dagger} \alpha_{-3 / 2}\right)+2 \alpha_{1 / 2}^{\dagger} \alpha_{-1 / 2} \\
\left(S^{+}\right)^{2} & =2 \sqrt{3}\left(\alpha_{3 / 2}^{\dagger} \alpha_{-1 / 2}+\alpha_{1 / 2}^{\dagger} \alpha_{-3 / 2}\right)
\end{aligned}
$$

Next, we apply an $\mathrm{SU}(4)$ rotation in the space of $\alpha_{m}^{\dagger}$ bosons:

$$
\begin{aligned}
& a_{A}^{\dagger}=a_{0, A}^{\dagger}=\frac{1}{\sqrt{6 \eta^{2}+2}}\left[e^{\frac{3}{2} i \varphi_{A}} \alpha_{-3 / 2}^{\dagger}+e^{-\frac{3}{2} i \varphi_{A}} \alpha_{3 / 2}^{\dagger}+\sqrt{3} \eta\left(e^{\frac{1}{2} i \varphi_{A}} \alpha_{-1 / 2}^{\dagger}+e^{-\frac{1}{2} i \varphi_{A}} \alpha_{1 / 2}^{\dagger}\right)\right], \\
& b_{A}^{\dagger}=a_{1, A}^{\dagger}=\frac{1}{\sqrt{14 \eta^{2}-8 \eta+2}}\left[\sqrt{3} \eta\left(e^{\frac{3}{2} i \varphi_{A}} \alpha_{-3 / 2}^{\dagger}-e^{-\frac{3}{2} i \varphi_{A}} \alpha_{3 / 2}^{\dagger}\right)+(2 \eta-1)\left(e^{\frac{1}{2} i \varphi_{A}} \alpha_{-1 / 2}^{\dagger}-e^{-\frac{1}{2} i \varphi_{A}} \alpha_{1 / 2}^{\dagger}\right)\right], \\
& c_{A}^{\dagger}=a_{2, A}^{\dagger}=\frac{1}{\sqrt{6 \eta^{2}+2}}\left[\sqrt{3} \eta\left(e^{\frac{3}{2} i \varphi_{A}} \alpha_{-3 / 2}^{\dagger}+e^{-\frac{3}{2} i \varphi_{A}} \alpha_{3 / 2}^{\dagger}\right)-\left(e^{\frac{1}{2} i \varphi_{A}} \alpha_{-1 / 2}^{\dagger}+e^{-\frac{1}{2} i \varphi_{A}} \alpha_{1 / 2}^{\dagger}\right)\right], \\
& d_{A}^{\dagger}=a_{3, A}^{\dagger}=\frac{1}{\sqrt{14 \eta^{2}-8 \eta+2}}\left[(2 \eta-1)\left(e^{\frac{3}{2} i \varphi_{A}} \alpha_{-3 / 2}^{\dagger}-e^{-\frac{3}{2} i \varphi_{A}} \alpha_{3 / 2}^{\dagger}\right)-\sqrt{3} \eta\left(e^{\frac{1}{2} i \varphi_{A}} \alpha_{-1 / 2}^{\dagger}-e^{-\frac{1}{2} i \varphi_{A}} \alpha_{1 / 2}^{\dagger}\right)\right],
\end{aligned}
$$

and analogous expressions hold for the bosons on the $B$ sites.

\section{Variational solution}

In the rotated basis the variational wave functionn corresponds to the $a_{A}^{\dagger} \mid$ vacuum $\rangle$. The energy per site, as a function of $\eta$ and $\varphi=\varphi_{A}=-\varphi_{B}$, reads

$$
\begin{aligned}
\frac{E(\eta, \varphi)}{N}= & \frac{3}{4} \frac{\left(\eta^{2}+3\right)}{\left(3 \eta^{2}+1\right)} \Lambda+\frac{18 \eta^{2}(\eta+1)^{2}}{\left(3 \eta^{2}+1\right)^{2}} J \cos 2 \varphi \\
& -\frac{3 \eta(\eta+1)}{3 \eta^{2}+1} g_{x x} h_{x} \cos \varphi
\end{aligned}
$$

Minimizing the $E(\eta, \varphi)$ with respect to variational parameters $\eta$ and $\varphi$, we get two solutions: (i) canted Néelstate, defined via the following set of equations:

$$
\begin{aligned}
\Lambda & =\frac{3(3 \eta+1)\left(\eta^{2}-1\right)}{3 \eta^{2}+1} J, \\
g_{x x} h_{x} & =\frac{24 \eta(\eta+1)}{\left(3 \eta^{2}+1\right)} J \cos \varphi .
\end{aligned}
$$

The spins cant in the direction of the field keeping the $\eta$ parameter unchanged. The limiting cases for $\eta$ are

$$
\eta= \begin{cases}1+\frac{\Lambda}{6 J}+O\left(\Lambda^{2} / J^{2}\right), & \text { if } \Lambda \ll J \\ \frac{\Lambda}{3 J}-\frac{1}{3}+O(J / \Lambda), & \text { if } \Lambda \gg J\end{cases}
$$

(ii) For high enough magnetic field, the spins in the A and B sublattice become equal and parallel to the field, setting $\varphi=0$, and $\eta$ is obtained from:

$$
\Lambda=\frac{(\eta-1)(3 \eta+1)}{4 \eta} g_{x x} h_{x}-\frac{3(3 \eta+1)\left(\eta^{2}-1\right)}{3 \eta^{2}+1} J .
$$

$\eta \rightarrow 1$ as the field $h_{x} \rightarrow \infty$.

While the usual procedure is to solve the equations for $\eta$ and $\varphi$, we prefer to express the $\Lambda$ as a function of $\eta$ in the following. 


\section{Multiboson spin-waves}

The $b_{X}, c_{X}$, and $d_{X}$ (i.e. $a_{\nu, X}$, with $X=A, B$ ) bosons in Eqs. (7b) - (7dd) take the role of the Holstein-Primakoff bosons. After the $1 / M$ expansion, the spin dipole operators are

$$
\begin{aligned}
S_{X}^{x}= & M \frac{3 \eta(\eta+1)}{3 \eta^{2}+1} \cos \varphi_{X} \\
& +\sqrt{M}\left[-\frac{i \sqrt{3} \sqrt{7 \eta^{2}-4 \eta+1}}{2 \sqrt{3 \eta^{2}+1}} \sin \varphi_{X}\left(b_{X}^{\dagger}-b_{X}\right)+\frac{\sqrt{3}(\eta-1)(3 \eta+1)}{2\left(3 \eta^{2}+1\right)} \cos \varphi_{X}\left(c_{X}^{\dagger}+c_{X}\right)\right], \\
S_{X}^{y}= & M \frac{3 \eta(\eta+1)}{3 \eta^{2}+1} \sin \varphi_{X} \\
& +\sqrt{M}\left[\frac{i \sqrt{3} \sqrt{7 \eta^{2}-4 \eta+1}}{2 \sqrt{3 \eta^{2}+1}} \cos \varphi_{X}\left(b_{X}^{\dagger}-b_{X}\right)+\frac{\sqrt{3}(\eta-1)(3 \eta+1)}{2\left(3 \eta^{2}+1\right)} \sin \varphi_{X}\left(c_{X}^{\dagger}+c_{X}\right)\right], \\
S_{X}^{z}= & \sqrt{M}\left[-\frac{\sqrt{3} \eta(\eta+1)}{\sqrt{3 \eta^{2}+1} \sqrt{7 \eta^{2}-4 \eta+1}}\left(b_{X}^{\dagger}+b_{X}\right)+\frac{3(\eta-1)^{2}}{2 \sqrt{3 \eta^{2}+1} \sqrt{7 \eta^{2}-4 \eta+1}}\left(d_{X}^{\dagger}+d_{X}\right)\right],
\end{aligned}
$$

while the spin quadrupole operators are

$$
\begin{aligned}
& \left(S_{X}^{z}\right)^{2}=M \frac{3\left(\eta^{2}+3\right)}{4\left(3 \eta^{2}+1\right)}+\frac{2 \sqrt{3} \eta}{3 \eta^{2}+1} \sqrt{M}\left(c_{X}^{\dagger}+c_{X}\right), \\
& \left(S_{X}^{x}\right)^{2}-\left(S_{X}^{y}\right)^{2}=M \frac{6 \eta}{3 \eta^{2}+1} \cos 2 \varphi_{X} \\
& +\left[-\frac{i \sqrt{3}(\eta+1)(3 \eta-1)}{\sqrt{3 \eta^{2}+1} \sqrt{7 \eta^{2}-4 \eta+1}} \sin 2 \varphi_{X}\left(b_{X}^{\dagger}-b_{X}\right)+\frac{\sqrt{3}\left(3 \eta^{2}-1\right)}{3 \eta^{2}+1} \cos 2 \varphi_{X}\left(c_{X}^{\dagger}+c_{X}\right)\right. \\
& \left.-\frac{6 i(\eta-1) \eta}{\sqrt{3 \eta^{2}+1} \sqrt{7 \eta^{2}-4 \eta+1}} \sin 2 \varphi_{X}\left(d_{X}^{\dagger}-d_{X}\right)\right] \sqrt{M} \\
& S_{X}^{x} S_{X}^{y}+S_{X}^{y} S_{X}^{x}=M \frac{6 \eta}{3 \eta^{2}+1} \sin 2 \varphi_{X} \\
& +\left[\frac{i \sqrt{3}(\eta+1)(3 \eta-1)}{\sqrt{3 \eta^{2}+1} \sqrt{7 \eta^{2}-4 \eta+1}} \cos 2 \varphi_{X}\left(b_{X}^{\dagger}-b_{X}\right)+\frac{\sqrt{3}\left(3 \eta^{2}-1\right)}{3 \eta^{2}+1} \sin 2 \varphi_{X}\left(c_{X}^{\dagger}+c_{X}\right)\right. \\
& \left.+\frac{6 i(\eta-1) \eta}{\sqrt{3 \eta^{2}+1} \sqrt{7 \eta^{2}-4 \eta+1}} \cos 2 \varphi_{X}\left(d_{X}^{\dagger}-d_{X}\right)\right] \sqrt{M} \\
& S_{X}^{x} S_{X}^{z}+S_{X}^{z} S_{X}^{x}=\sqrt{M}\left[-\frac{\sqrt{3}(\eta+1)(3 \eta-1)}{\sqrt{3 \eta^{2}+1} \sqrt{7 \eta^{2}-4 \eta+1}} \cos \varphi_{X}\left(b_{X}^{\dagger}+b_{X}\right)+i \sqrt{3} \sin \varphi_{X}\left(c_{X}^{\dagger}-c_{X}\right)\right. \\
& \left.-\frac{6(\eta-1) \eta}{\sqrt{3 \eta^{2}+1} \sqrt{7 \eta^{2}-4 \eta+1}} \cos \varphi_{X}\left(d_{X}^{\dagger}+d_{X}\right)\right] \\
& S_{X}^{y} S_{X}^{z}+S_{X}^{z} S_{X}^{y}=\sqrt{M}\left[-\frac{\sqrt{3}(\eta+1)(3 \eta-1)}{\sqrt{3 \eta^{2}+1} \sqrt{7 \eta^{2}-4 \eta+1}} \sin \varphi_{X}\left(b_{X}^{\dagger}+b_{X}\right)-i \sqrt{3} \cos \varphi_{X}\left(c_{X}^{\dagger}-c_{X}\right)\right. \\
& \left.-\frac{6(\eta-1) \eta d_{X}}{\sqrt{3 \eta^{2}+1} \sqrt{7 \eta^{2}-4 \eta+1}} \sin \varphi_{X}\left(d_{X}^{\dagger}+d_{X}\right)\right]
\end{aligned}
$$

where terms that are proportional to $M$ and $\sqrt{M}$ are shown.
The multiboson spin-wave Hamiltonian up to quadratic order in bosons reads:

$$
\mathcal{H} \approx M^{2} \mathcal{H}^{(0)}+M^{3 / 2} \mathcal{H}^{(1)}+M \mathcal{H}^{(2)}
$$


where $\mathcal{H}^{(0)}$ is equal to mean field energy $(\underline{8}), \mathcal{H}^{(1)}$ is identically zero when (9) is satisfied, and the quadratic term has the following form for the solution given by Eqs. (9):

$$
\begin{aligned}
\mathcal{H}^{(2)}= & +\frac{6(\eta+1)^{2}\left(9 \eta^{3}-5 \eta^{2}-\eta+1\right)}{\left(3 \eta^{2}+1\right)\left(7 \eta^{2}-4 \eta+1\right)} J\left(b_{A}^{\dagger} b_{A}+b_{B}^{\dagger} b_{B}\right)+\frac{72 \eta^{3}(\eta+1)^{2}}{\left(3 \eta^{2}+1\right)\left(7 \eta^{2}-4 \eta+1\right)} J\left(d_{A}^{\dagger} d_{A}+d_{B}^{\dagger} d_{B}\right) \\
& +\frac{9(\eta-1)^{4}}{\left(3 \eta^{2}+1\right)\left(7 \eta^{2}-4 \eta+1\right)} J_{z}\left(d_{A}^{\dagger} d_{B}^{\dagger}+d_{A} d_{B}+d_{A}^{\dagger} d_{B}+d_{B}^{\dagger} d_{A}\right) \\
& -\frac{6 \sqrt{3} \eta(\eta+1)(\eta-1)^{2}}{\left(3 \eta^{2}+1\right)\left(7 \eta^{2}-4 \eta+1\right)} J_{z}\left(d_{A} b_{B}+d_{B} b_{A}+b_{A}^{\dagger} d_{B}+b_{A}^{\dagger} d_{B}^{\dagger}+b_{B}^{\dagger} d_{A}+b_{B}^{\dagger} d_{A}^{\dagger}+d_{A}^{\dagger} b_{B}+d_{B}^{\dagger} b_{A}\right) \\
& +\frac{36 \sqrt{3} \eta^{2}(\eta+1)(\eta-1)^{2}}{\left(3 \eta^{2}+1\right)\left(7 \eta^{2}-4 \eta+1\right)} J\left(b_{A}^{\dagger} d_{A}+b_{B}^{\dagger} d_{B}+d_{A}^{\dagger} b_{A}+d_{B}^{\dagger} b_{B}\right) \\
& +\left[\frac{12 \eta^{2}(\eta+1)^{2}}{\left(3 \eta^{2}+1\right)\left(7 \eta^{2}-4 \eta+1\right)} J_{z}-\frac{3\left(7 \eta^{2}-4 \eta+1\right)}{3 \eta^{2}+1} J \cos 2 \varphi\right]\left(b_{A}^{\dagger} b_{B}^{\dagger}+b_{A} b_{B}+b_{A}^{\dagger} b_{B}+b_{B}^{\dagger} b_{A}\right) \\
& +\frac{3(3 \eta+1)(\eta-1) \sqrt{7 \eta^{2}-4 \eta+1}}{\left(3 \eta^{2}+1\right)^{3 / 2}} i \sin 2 \varphi\left(b_{A} c_{B}+b_{A} c_{B}^{\dagger}+b_{B}^{\dagger} c_{A}+b_{B}^{\dagger} c_{A}^{\dagger}-b_{A}^{\dagger} c_{B}-b_{A}^{\dagger} c_{B}^{\dagger}-b_{B} c_{A}-b_{B} c_{A}^{\dagger}\right) \\
& +\frac{3(3 \eta+1)^{2}(\eta-1)^{2}}{\left(3 \eta^{2}+1\right)^{2}} J \cos 2 \varphi\left(c_{A}^{\dagger} c_{B}^{\dagger}+c_{A} c_{B}+c_{A}^{\dagger} c_{B}+c_{A} c_{B}^{\dagger}\right)+6 J(\eta+1)\left(c_{A}^{\dagger} c_{A}+c_{B}^{\dagger} c_{B}\right) .
\end{aligned}
$$

We note that in zero field $(\varphi= \pm \pi / 2)$ and parallel spins the Hamiltonian separates into two parts, one involving $b$ and $d$ bosons, the other only the $c$ bosons. Similarly, for the uniform state in high fields, where the variational parameters are given by Eq. (11) we get $\mathcal{H}^{(2)}=\mathcal{H}_{b d}^{(2)}+$ $\mathcal{H}_{c}^{(2)}$ with

$$
\begin{aligned}
\mathcal{H}_{b d}^{(2)}= & \frac{9 J_{z}(\eta-1)^{4}}{\left(3 \eta^{2}+1\right)\left(7 \eta^{2}-4 \eta+1\right)}\left(d_{B} d_{A}+d_{A}^{\dagger} d_{B}+d_{A}^{\dagger} d_{B}^{\dagger}+d_{B}^{\dagger} d_{A}\right) \\
& -\frac{6 \sqrt{3} J_{z} \eta(\eta+1) d_{A} b_{B}(\eta-1)^{2}}{\left(3 \eta^{2}+1\right)\left(7 \eta^{2}-4 \eta+1\right)}\left(d_{A} b_{B}+d_{B} b_{A}+b_{A}^{\dagger} d_{B}+b_{A}^{\dagger} d_{B}^{\dagger}+b_{B}^{\dagger} d_{A}+b_{B}^{\dagger} d_{A}^{\dagger}+d_{A}^{\dagger} b_{B}+d_{B}^{\dagger} b_{A}\right) \\
& +\left(\frac{12 J_{z} \eta^{2}(\eta+1)^{2}}{\left(3 \eta^{2}+1\right)\left(7 \eta^{2}-4 \eta+1\right)}-\frac{3 J\left(7 \eta^{2}-4 \eta+1\right)}{3 \eta^{2}+1}\right)\left(b_{B} b_{A}+b_{A}^{\dagger} b_{B}^{\dagger}\right) \\
& +\left(\frac{12 J_{z} \eta^{2}(\eta+1)^{2}}{\left(3 \eta^{2}+1\right)\left(7 \eta^{2}-4 \eta+1\right)}+\frac{3 J\left(7 \eta^{2}-4 \eta+1\right)}{3 \eta^{2}+1}\right)\left(b_{A}^{\dagger} b_{B}+b_{B}^{\dagger} b_{A}\right) \\
& +\left(\frac{g_{x x} h_{x}(\eta+1)\left(9 \eta^{3}-5 \eta^{2}-\eta+1\right)}{2 \eta\left(7 \eta^{2}-4 \eta+1\right)}-\frac{6 J(\eta+1)^{2}\left(9 \eta^{3}-5 \eta^{2}-\eta+1\right)}{\left(3 \eta^{2}+1\right)\left(7 \eta^{2}-4 \eta+1\right)}\right)\left(b_{A}^{\dagger} b_{A}+b_{B}^{\dagger} b_{B}\right) \\
& +\left(\frac{3 \sqrt{3} g_{x x} h_{x}(\eta-1)^{2} \eta}{7 \eta^{2}-4 \eta+1}-\frac{36 \sqrt{3} J(\eta-1)^{2} \eta^{2}(\eta+1)}{\left(3 \eta^{2}+1\right)\left(7 \eta^{2}-4 \eta+1\right)}\right)\left(b_{A}^{\dagger} d_{A}+b_{B}^{\dagger} d_{B}+d_{A}^{\dagger} b_{A}+d_{B}^{\dagger} b_{B}\right) \\
& +\left(\frac{6 g_{x x} h_{x} \eta^{2}(\eta+1)}{7 \eta^{2}-4 \eta+1}-\frac{72 J \eta^{3}(\eta+1)^{2}}{\left(3 \eta^{2}+1\right)\left(7 \eta^{2}-4 \eta+1\right)}\right)\left(d_{A}^{\dagger} d_{A}+d_{B}^{\dagger} d_{B}\right)
\end{aligned}
$$

and

$$
\begin{aligned}
\mathcal{H}_{c}^{(2)}= & +\left(\frac{g_{x x} h_{x}\left(3 \eta^{2}+1\right)}{2 \eta}-6 J(\eta+1)\right)\left(c_{A}^{\dagger} c_{A}+c_{B}^{\dagger} c_{B}\right) \\
& +\frac{3 J(3 \eta+1)^{2}(\eta-1)^{2}}{\left(3 \eta^{2}+1\right)^{2}}\left(c_{B} c_{A}+c_{A}^{\dagger} c_{B}+c_{A}^{\dagger} c_{B}^{\dagger}+c_{B}^{\dagger} c_{A}\right) .
\end{aligned}
$$




\section{The spectrum in zero field}

The finite anisotropy reduces the symmetry down to $O(2)$, and the Goldstone mode associated with turning the order parameter in the $x y$ plane is desribed by the

$$
\begin{aligned}
\gamma^{\dagger}=\gamma \propto & 2 \eta(\eta+1)\left(b_{A}^{\dagger}+b_{B}^{\dagger}+b_{A}+b_{B}\right) \\
& -\sqrt{3}(\eta-1)^{2}\left(d_{A}^{\dagger}+d_{B}^{\dagger}+d_{A}+d_{B}\right)
\end{aligned}
$$

so that $\left[\mathcal{H}^{(2)}, \gamma^{\dagger}\right]=\left[\mathcal{H}^{(2)}, \gamma\right]=0$.
The energy of the remaining 5 modes: In the $d_{-}$mode the spin moves in the $x y$ plane and is of the same symmetry character as the Goldstone mode, with energy

$$
\omega_{d_{-}}=\frac{18(\eta+1) \sqrt{\eta\left(\eta^{3}-\eta^{2}+3 \eta+1\right)}}{3 \eta^{2}+1} J
$$

The energy of the $b_{+}$and $d_{+}$modes that depend on the $j_{z}=J_{z} / J$ is given by the

$$
\frac{\omega^{4}}{J^{4}}=\left(\frac{6 \eta+6}{3 \eta^{2}+1}\right)^{2}\left(9 \eta^{4}+9 \eta^{3}+17 \eta^{2}+7 \eta+2-8 \eta^{2} j_{z}\right) \frac{\omega^{2}}{J^{2}}-72 \eta^{3}\left(\frac{6 \eta+6}{3 \eta^{2}+1}\right)^{4}\left(4 \eta^{3}-\eta^{3} j_{z}+\eta^{2} j_{z}-3 \eta j_{z}-j_{z}\right)(1
$$

equation. The four aforementioned modes consist of $b$ and $d$ bosons.

Finally, the two "stretching" modes that involve the $c$ bosons only:

$$
\begin{aligned}
& \omega_{c_{-}}=\frac{6 \sqrt{(\eta+1)\left(9 \eta^{5}+18 \eta^{4}-6 \eta^{3}+4 \eta^{2}+5 \eta+2\right)}}{3 \eta^{2}+1} J \\
& \omega_{c_{+}}=\frac{6 \sqrt{\eta(\eta+1)\left(9 \eta^{4}+18 \eta^{2}+8 \eta-3\right)}}{3 \eta^{2}+1} J
\end{aligned}
$$

In the limit of small $\Lambda$ the energies are

$$
\begin{aligned}
& \omega_{b_{-}}=0, \\
& \omega_{b_{+}}^{2}=24 J\left[3\left(J-J_{z}\right)+\Lambda\right]+O\left(\Lambda^{2}\right), \\
& \omega_{c_{-}}=12 J+\Lambda+\frac{1}{8} \frac{\Lambda^{2}}{J}+O\left(\Lambda^{3} / J^{2}\right), \\
& \omega_{c_{+}}=12 J+\Lambda-\frac{1}{24} \frac{\Lambda^{2}}{J}+O\left(\Lambda^{3} / J^{2}\right), \\
& \omega_{d_{-}}=18 J+O\left(\Lambda^{3} / J^{2}\right), \\
& \omega_{d_{+}}=18 J+O\left(\Lambda^{3} / J^{2}\right) .
\end{aligned}
$$

The $\omega_{b_{+}}$shows the typical square-root behaviour of the anisotropy gap on the exchange anysotropy $J-J_{z}$ and single-ion anisotropy $\Lambda$ for small gap.

In the limit of large single-ion anisotropy $\left(\Lambda \gg J, J_{z}\right)$ :

$$
\begin{aligned}
& \omega_{b_{-}}=0 \\
& \omega_{b_{+}}^{2}=32 J\left(4 J-J_{z}+12 J^{2} / \Lambda\right)+O\left(1 / \Lambda^{2}\right), \\
& \omega_{c_{-}}=2 \Lambda+7 J+O\left(J^{2} / \Lambda\right) \\
& \omega_{c_{+}}=2 \Lambda+J+O\left(J^{2} / \Lambda\right) \\
& \omega_{d_{-}}=2 \Lambda+J+O\left(J^{2} / \Lambda\right) \\
& \omega_{d_{+}}=2 \Lambda+7 J+O\left(J^{2} / \Lambda\right)
\end{aligned}
$$

